\title{
The Applications of Functional Variants of Jensen's Inequality
}

\author{
Zlatko Pavić \\ Mechanical Engineering Faculty in Slavonski Brod, University of Osijek, Trg Ivane Brlić Mažuranić 2, 35000 Slavonski Brod, Croatia \\ Correspondence should be addressed to Zlatko Pavić; zpavic@sfsb.hr \\ Received 31 May 2013; Accepted 2 September 2013 \\ Academic Editor: Eva A. Gallardo Gutiérrez \\ Copyright (C) 2013 Zlatko Pavić. This is an open access article distributed under the Creative Commons Attribution License, which \\ permits unrestricted use, distribution, and reproduction in any medium, provided the original work is properly cited. \\ The paper is inspired by McShane's results on the functional form of Jensen's inequality for convex functions of several variables. \\ The work is focused on applications and generalizations of this important result. At that, the generalizations of Jensen's inequality \\ are obtained using the positive linear functionals.
}

\section{Introduction}

1.1. Vector Space of Real Valued Functions and Positive Linear Functionals. Given a nonempty set $\mathscr{X}$ we consider a space of functions $g: X \rightarrow \mathbb{R}$ containing the unit function $g=1$ defined with $1(x)=1$ for every $x \in \mathscr{X}$. Let $\mathbb{X}$ be a real vector space of real valued functions on $\mathscr{X}$ containing the unit function. A linear functional $M: \mathbb{X} \rightarrow \mathbb{R}$ is said to be positive (nonnegative) or monotone if $M(g) \geq 0$ for every nonnegative function $g \in \mathbb{X}$. If $M(1)=1$, we say that the functional $M$ is unital or normalized.

1.2. Functional Forms of Jensen's Inequality. In 1931, Jessen [1] formulated the functional form of Jensen's inequality for convex functions of one variable.

Theorem A. Let $\mathscr{I} \subseteq \mathbb{R}$ be a closed interval. Let $g \in \mathbb{X}$ be a function such that $g(x) \in \mathscr{I}$ for every $x \in X$. Let $f: \mathscr{I} \rightarrow \mathbb{R}$ be a continuous convex function such that $f(g) \in \mathbb{X}$.

Then every unital positive linear functional $M: \mathbb{X} \rightarrow \mathbb{R}$ satisfies the inequality

$$
f(M(g)) \leq M(f(g)) .
$$

The interval $\mathscr{I}$ must be closed; otherwise, it could happen that $M(g) \notin \mathscr{I}$ as mentioned in [2].

Example 1. Let $\mathscr{X}=\mathscr{I}=\langle 0,1], \mathbb{X}=\{g:\langle 0,1] \rightarrow \mathbb{R} \mid$ $\left.\lim _{x \rightarrow 0_{+}} g(x) \in \mathbb{R}\right\}$, and $M: \mathbb{X} \rightarrow \mathbb{R}$ is defined by $M(g)=$ $\lim _{x \rightarrow 0+} g(x)$. Take the identity function $g(x)=x$ for $x \in \mathscr{I}$. Then $g \in \mathbb{X}$ and $g(x) \in \mathscr{I}$ for every $x \in \mathscr{X}$, but $M(g)=0 \notin \mathscr{I}$.
The function $f$ must be continuous; otherwise, it could happen that the inequality in (1) does not apply as noted in [3].

Example 2. Consider the previous example with $\mathscr{X}=\mathscr{I}=$ $[0,1]$. Take the convex function $f:[0,1] \rightarrow \mathbb{R}$ defined by $f(0)=1$ and $f(x)=0$ for $x \in\langle 0,1]$. Then $f(M(g))=f(0)=$ $1>0=M(f)=M(f(g))$.

In 1937, McShane [4, Theorems 1-2] extended the functional form of Jensen's inequality to convex functions of several variables. He has covered the generalization in two steps, calling them the geometric (Theorem B) and analytic (Theorem C) formulation of Jensen's inequality.

Theorem B. Let $\mathscr{C} \subseteq \mathbb{R}^{n}$ be a closed convex set. Let $g_{i} \in \mathbb{X}$ be functions such that $\left(g_{1}(x), \ldots, g_{n}(x)\right) \in \mathscr{C}$ for every $x \in \mathscr{X}$.

Then every unital positive linear functional $M: \mathbb{X} \rightarrow \mathbb{R}$ satisfies the inclusion

$$
\left(M\left(g_{1}\right), \ldots, M\left(g_{n}\right)\right) \in \mathscr{C} .
$$

Theorem C. Let $\mathscr{C} \subseteq \mathbb{R}^{n}$ be a closed convex set. Let $g_{i} \in \mathbb{X}$ be functions such that $\left(g_{1}(x), \ldots, g_{n}(x)\right) \in \mathscr{C}$ for every $x \in \mathscr{X}$. Let $f: \mathscr{C} \rightarrow \mathbb{R}$ be a continuous convex function such that $f\left(g_{1}\right.$, $\left.\ldots, g_{n}\right) \in \mathbb{X}$.

Then every unital positive linear functional $M: \mathbb{X} \rightarrow \mathbb{R}$ satisfies the inequality

$$
f\left(M\left(g_{1}\right), \ldots, M\left(g_{n}\right)\right) \leq M\left(f\left(g_{1}, \ldots, g_{n}\right)\right)
$$


If the function $f$ is concave, then the reverse inequality is valid in (3). The hyperplanes that contain the set $\mathscr{C}$ were used in the proof of Theorem B. The epigraph of the function $f$ :

$$
\begin{gathered}
\operatorname{epi}(f)=\left\{\left(x_{1}, \ldots, x_{n}, x_{n+1}\right) \in \mathscr{C} \times \mathbb{R} \mid x_{n+1}\right. \\
\left.\geq f\left(x_{1}, \ldots, x_{n}\right)\right\}
\end{gathered}
$$

and Theorem B were applied in the proof of Theorem C.

\section{The Applications and Generalizations of McShane's Result}

This section presents general applications and generalizations of Theorem C. The main generalization is Theorem 8 .

Using McShane's inequality in (3) it is very easy to get the functional form of Hölder's and Minkowski's inequality for $n$ nonnegative functions.

Corollary 3 (the functional form of Hölder's inequality). Let $g_{i}: X \rightarrow \mathbb{R}$ be nonnegative functions such that $g_{1} \cdots g_{n} \in \mathbb{X}$. Let $p_{i}>1$ be real numbers such that $\sum_{i=1}^{n} 1 / p_{i}=1$ and all $g_{i}^{p_{i}} \in$ X.

Then every unital positive linear functional $M: \mathbb{X} \rightarrow \mathbb{R}$ satisfies the inequality

$$
M\left(g_{1} \cdots g_{n}\right) \leq\left(M\left(g_{1}^{p_{1}}\right)\right)^{1 / p_{1}} \cdots\left(M\left(g_{n}^{p_{n}}\right)\right)^{1 / p_{n}} .
$$

Proof. Applying the concave function $f\left(x_{1}, \ldots, x_{n}\right)=x_{1}^{1 / p_{1}}$ $\cdots x_{n}^{1 / p_{n}}$, where all $x_{i} \geq 0$, together with the functions $g_{i}^{p_{i}}$ to the reverse inequality in (3), it follows that

$$
\left(M\left(g_{1}^{p_{1}}\right)\right)^{1 / p_{1}} \cdots\left(M\left(g_{n}^{p_{n}}\right)\right)^{1 / p_{n}} \geq M\left(g_{1} \cdots g_{n}\right),
$$

which is the required inequality.

Corollary 4 (the functional form of Minkowski's inequality). Let $g_{i}: X \rightarrow \mathbb{R}$ be nonnegative functions for $i=1, \ldots, n$. Let $p \geq 1$ be a real number such that $\left(g_{1}+\cdots+g_{n}\right)^{p} \in \mathbb{X}$ and all $g_{i}^{p} \in \mathbb{X}$.

Then every unital positive linear functional $M: \mathbb{X} \rightarrow \mathbb{R}$ satisfies the inequality

$$
\begin{aligned}
& \left(M\left(\left(g_{1}+\cdots+g_{n}\right)^{p}\right)\right)^{1 / p} \\
& \quad \leq\left(M\left(g_{1}^{p}\right)\right)^{1 / p}+\cdots+\left(M\left(g_{n}^{p}\right)\right)^{1 / p} .
\end{aligned}
$$

Proof. Applying the concave function $f\left(x_{1}, \ldots, x_{n}\right)=\left(x_{1}^{1 / p}+\right.$ $\left.\cdots+x_{n}^{1 / p}\right)^{p}$, where all $x_{i} \geq 0$, and the functions $g_{i}^{p}$ to the reverse inequality in (3), it follows that

$$
\begin{gathered}
\left(\left(M\left(g_{1}^{p}\right)\right)^{1 / p}+\cdots+\left(M\left(g_{n}^{p}\right)\right)^{1 / p}\right)^{p} \\
\geq M\left(\left(g_{1}+\cdots+g_{n}\right)^{p}\right)
\end{gathered}
$$

and rising to the power of $1 / p$ gives the desired inequality.
The McShane inequality in (3) can be easily generalized by introducing the weighted function $p \in \mathbb{X}$ as follows.

Theorem 5. Let $\mathscr{C} \subseteq \mathbb{R}^{n}$ be a closed convex set. Let $g_{i}: \mathscr{X} \rightarrow$ $\mathbb{R}$ be functions such that $\left(g_{1}(x), \ldots, g_{n}(x)\right) \in \mathscr{C}$ for every $x \in$ $\mathscr{X}$. Let $p \in \mathbb{X}$ be either a nonnegative or nonpositive function such that all $p g_{i} \in \mathbb{X}$. Let $f: \mathscr{C} \rightarrow \mathbb{R}$ be a continuous convex function such that $p f\left(g_{1}, \ldots, g_{n}\right) \in \mathbb{X}$.

Then every positive linear functional $M: \mathbb{X} \rightarrow \mathbb{R}$ such that $M(p) \neq 0$ satisfies the inequality

$$
f\left(\frac{M\left(p g_{1}\right)}{M(p)}, \ldots, \frac{M\left(p g_{n}\right)}{M(p)}\right) \leq \frac{M\left(p f\left(g_{1}, \ldots, g_{n}\right)\right)}{M(p)} .
$$

Proof. We use the function vector space $\mathbb{Y}=\{g: \mathscr{X} \rightarrow \mathbb{R} \mid$ $p g \in \mathbb{X}\}$ and the linear functional $L: \mathbb{Y} \rightarrow \mathbb{R}$ defined with $L(g)=M(p g) / M(p)$. The functional $L$ is positive and unital. Since all $g_{i} \in \mathbb{Y}$ and $f\left(g_{1}, \ldots, g_{n}\right) \in \mathbb{Y}$, the functional values $L\left(g_{i}\right)$ and $L\left(f\left(g_{1}, \ldots, g_{n}\right)\right)$ may be applied to the inequality in (3).

If $\mathbb{X}$ is a vector space of real valued $\mu$-integrable functions on $\mathscr{C}$, then using the inequality in (9) with the positive linear functional

$$
M(g)=\int_{\mathscr{C}} g d \mu
$$

we get the following integral form of Jensen's inequality for convex functions of several variables.

Corollary 6. Let $\mu$ be a measure on a closed convex set $\mathscr{C} \subseteq$ $\mathbb{R}^{n}$. Let $g_{i}: \mathscr{C} \rightarrow \mathbb{R}$ be $\mu$-measurable functions such that $\left(g_{1}(x), \ldots, g_{n}(x)\right) \in \mathscr{C}$ for every $x \in \mathscr{C}$. Let $p: \mathscr{C} \rightarrow \mathbb{R}$ be either a nonnegative or nonpositive $\mu$-integrable function such that $\int_{\mathscr{C}} p d \mu \neq 0$ and all $p g_{i}$ are $\mu$-integrable.

Then every continuous convex function $f: \mathscr{C} \rightarrow \mathbb{R}$ such that $p f\left(g_{1}, \ldots, g_{n}\right)$ is $\mu$-integrable satisfies the integral inequality

$$
f\left(\frac{\int_{\mathscr{C}} p g_{1} d \mu}{\int_{\mathscr{C}} p d \mu}, \ldots, \frac{\int_{\mathscr{C}} p g_{n} d \mu}{\int_{\mathscr{C}} p d \mu}\right) \leq \frac{\int_{\mathscr{C}} p f\left(g_{1}, \ldots, g_{n}\right) d \mu}{\int_{\mathscr{C}} p d \mu}
$$

Choosing points $\left(x_{j 1}, \ldots, x_{j n}\right) \in \mathscr{C}$ for $j=1, \ldots, m$ and applying the inequality in (9) with the positive linear functional

$$
M(g)=\sum_{j=1}^{m} g\left(x_{j 1}, \ldots, x_{j n}\right)
$$

projections $g_{i}\left(x_{j 1}, \ldots, x_{j n}\right)=x_{j i}$, and weighted function $p\left(x_{j 1}, \ldots, x_{j n}\right)=\alpha_{j}$, we have the following discrete form of Jensen's inequality for convex functions of several variables.

Corollary 7. Let $\mathscr{C} \subseteq \mathbb{R}^{n}$ be a closed convex set. Let $\left(x_{j 1}\right.$, $\left.\ldots, x_{j n}\right) \in \mathscr{C}$ be points for $j=1, \ldots, m$. Let $\alpha_{j} \in \mathbb{R}$ be either nonnegative or nonpositive coefficients such that $\sum_{j=1}^{m} \alpha_{j} \neq 0$. 
Then every continuous convex function $f: \mathscr{C} \rightarrow \mathbb{R}$ satisfies the discrete inequality

$$
f\left(\frac{\sum_{j=1}^{m} \alpha_{j} x_{j 1}}{\sum_{j=1}^{m} \alpha_{j}}, \ldots, \frac{\sum_{j=1}^{m} \alpha_{j} x_{j n}}{\sum_{j=1}^{m} \alpha_{j}}\right) \leq \frac{\sum_{j=1}^{m} \alpha_{j} f\left(x_{j 1}, \ldots, x_{j n}\right)}{\sum_{j=1}^{m} \alpha_{j}} .
$$

Now we use $m$ nonempty sets $\mathscr{X}_{j}$ and associated function vector spaces $\mathbb{X}_{j}$ containing the unit function.

Theorem 8. Let $\mathscr{C} \subseteq \mathbb{R}^{n}$ be a closed convex set. Let $g_{j i}: \mathscr{X}_{j} \rightarrow$ $\mathbb{R}$ be functions such that $\left(g_{j 1}(x), \ldots, g_{j n}(x)\right) \in \mathscr{C}$ for every $x \in \mathscr{X}_{j}$ for $j=1, \ldots, m$. Let $p_{j} \in \mathbb{X}_{j}$ be either nonnegative or nonpositive functions such that $p_{j} g_{j i} \in \mathbb{X}_{j}$. Let $f: \mathscr{C} \rightarrow \mathbb{R}$ be a continuous convex function such that $p_{j} f\left(g_{j 1}, \ldots, g_{j n}\right) \in \mathbb{X}_{j}$.

Then every $m$-tuple of positive linear functionals $M_{j}$ : $\mathbb{X}_{j} \rightarrow \mathbb{R}$ such that all $M_{j}\left(p_{j}\right) \neq 0$ satisfies the inequality

$$
\begin{gathered}
f\left(\frac{\sum_{j=1}^{m} M_{j}\left(p_{j} g_{j 1}\right)}{\sum_{j=1}^{m} M_{j}\left(p_{j}\right)}, \ldots, \frac{\sum_{j=1}^{m} M_{j}\left(p_{j} g_{j n}\right)}{\sum_{j=1}^{m} M_{j}\left(p_{j}\right)}\right) \\
\leq \frac{\sum_{j=1}^{m} M_{j}\left(p_{j} f\left(g_{j 1}, \ldots, g_{j n}\right)\right)}{\sum_{j=1}^{m} M_{j}\left(p_{j}\right)} .
\end{gathered}
$$

Proof. First we apply the inequality in (13) to the numbers $x_{j i}=M_{j}\left(p_{j} g_{j i}\right) / M_{j}\left(p_{j}\right)$ and coefficients $\alpha_{j}=M_{j}\left(p_{j}\right)$ then the inequality in (9) to every $M_{j}$ :

$$
\begin{aligned}
& f\left(\frac{\sum_{j=1}^{m} M_{j}\left(p_{j} g_{j 1}\right)}{\sum_{j=1}^{m} M_{j}\left(p_{j}\right)}, \ldots, \frac{\sum_{j=1}^{m} M_{j}\left(p_{j} g_{j n}\right)}{\sum_{j=1}^{m} M_{j}\left(p_{j}\right)}\right) \\
& =f\left(\frac{\sum_{j=1}^{m} M_{j}\left(p_{j}\right)\left(M_{j}\left(p_{j} g_{j 1}\right) / M_{j}\left(p_{j}\right)\right)}{\sum_{j=1}^{m} M_{j}\left(p_{j}\right)}, \ldots,\right. \\
& \leq \sum_{j=1}^{m} M_{j}\left(p_{j}\right) f\left(\frac{M_{j}\left(p_{j} g_{j 1}\right)}{M_{j}\left(p_{j}\right)}, \ldots, \frac{M_{j}\left(p_{j} g_{j n}\right)}{M_{j}\left(p_{j}\right)}\right) \\
& \left.\quad \times\left(\sum_{j=1}^{m} M_{j}\left(p_{j}\right)\right)^{-1} \leq \frac{\left.\sum_{j=1}^{m} M_{j}\left(p_{j} g_{j n}\right) / M_{j}\left(p_{j}\right)\right)}{\sum_{j=1}^{m} M_{j}\left(g_{j 1}, \ldots, g_{j n}\right)}\right)
\end{aligned}
$$

and so we get the inequality in (14).

The inequality in (14) covers all the inequalities in this section.

\section{The Generalizations of Jensen's Inequality Using Functionals}

This section presents the actual results of the paper. The main results are the generalizations of Jensen's inequality written in Theorem 12 for convex functions of one variable and Theorem 16 for convex functions of several variables.
3.1. Inequalities for Convex Functions with One Variable. In what follows, we use a bounded interval $[a, b] \subset \mathbb{R}$ assuming $a<b$. Every $x \in \mathbb{R}$ can be uniquely presented as the affine combination

$$
x=\alpha_{x} a+\beta_{x} b
$$

where

$$
\alpha_{x}=\frac{\left|\begin{array}{ll}
x & 1 \\
b & 1
\end{array}\right|}{\left|\begin{array}{ll}
a & 1 \\
b & 1
\end{array}\right|}, \quad \beta_{x}=-\frac{\left|\begin{array}{ll}
x & 1 \\
a & 1
\end{array}\right|}{\left|\begin{array}{ll}
a & 1 \\
b & 1
\end{array}\right|} .
$$

Lemma 9. Let $c \in[a, b]$ be a point. Let $\alpha, \beta \in[0,1]$ and $\gamma \in$ $[-1,1]$ be coefficients of the sum $\alpha+\beta+\gamma=1$.

Then the affine combination $\alpha a+\beta b+\gamma c$ belongs to $[a, b]$.

Proof. Since $c \in[a, b]$, it has to be $c=\alpha_{c} a+\beta_{c} b$ for coefficients $\alpha_{c}$ and $\beta_{c}$ taken from the formulas in (17). Then we have

$$
\begin{aligned}
\alpha a & +\beta b+\gamma c \\
& =\alpha a+\beta b+(1-\alpha-\beta)\left(\alpha_{c} a+\beta_{c} b\right) \\
& =\left[\alpha\left(1-\alpha_{c}\right)+(1-\beta) \alpha_{c}\right] a+\left[\beta\left(1-\beta_{c}\right)+(1-\alpha) \beta_{c}\right] b .
\end{aligned}
$$

The coefficients in the square brackets are nonnegative with the sum equal to 1 , so the observed expression $\alpha a+\beta b+\gamma c$ belongs to $[a, b]$.

Sufficient conditions on the coefficients in Lemma 9 are $\alpha, \beta \in[0,1]$ and $\alpha+\beta+\gamma=1$. Applying these conditions, it follows that $\gamma \in[-1,1]$.

Theorem 10. Let $c_{j} \in[a, b]$ be points for $j=1, \ldots, m$. Let $\alpha, \beta, \gamma_{j} \in[0,1]$ and $\gamma \in[-1,1]$ be coefficients of the sums $\alpha+\beta+\gamma=\sum_{j=1}^{m} \gamma_{j}=1$.

Then the affine combination $\alpha a+\beta b+\gamma \sum_{j=1}^{m} \gamma_{j} c_{j} \in[a, b]$.

Proof. Since $\sum_{j=1}^{m} \gamma_{j} c_{j} \in[a, b]$, the affine combination $\alpha a+$ $\beta b+\gamma \sum_{j=1}^{m} \gamma_{j} c_{j} \in[a, b]$ by Lemma 9 .

Corollary 11. Let $g:[a, b] \rightarrow \mathbb{R}$ be a monotone function, and let $x_{j} \in[a, b]$ be points for $j=1, \ldots, m$. Let $\alpha, \beta, \gamma_{j} \in$ $[0,1]$ and $\gamma \in[-1,1]$ be coefficients of the sums $\alpha+\beta+\gamma=$ $\sum_{j=1}^{m} \gamma_{j}=1$.

Then the affine combination $\alpha g(a)+\beta g(b)+$ $\gamma \sum_{j=1}^{m} \gamma_{j} g\left(x_{j}\right) \in \operatorname{conv}\{g(a), g(b)\}$.

The convex hull of a set $\mathcal{S}$ will be denoted by $\operatorname{conv} \mathcal{S}$.

Theorem 12. Let $g:[a, b] \rightarrow \mathbb{R}$ be a monotone function, and let $x_{j} \in[a, b]$ be points for $j=1, \ldots, m$. Let $\alpha, \beta, \gamma_{j} \in[0,1]$ and $\gamma \in[-1,1]$ be coefficients of the sums $\alpha+\beta+\gamma=\sum_{j=1}^{m} \gamma_{j}=1$.

Then every continuous convex function $f: \operatorname{conv}\{g(a)$, $g(b)\} \rightarrow \mathbb{R}$ satisfies the inequality

$$
\begin{aligned}
& f\left(\alpha g(a)+\beta g(b)+\gamma \sum_{j=1}^{m} \gamma_{j} g\left(x_{j}\right)\right) \\
& \quad \leq \alpha f(g(a))+\beta f(g(b))+\gamma \sum_{j=1}^{m} \gamma_{j} f\left(g\left(x_{j}\right)\right) .
\end{aligned}
$$


Proof. If $f$ is monotone, then the Jensen inequality in (1) should be applied to the function space $\mathbb{X}$ generated with $g$ and $f(g)$, that is,

$$
\mathbb{X}=\left\{h:[a, b] \longrightarrow \mathbb{R} \mid h=c_{1} g+c_{2} f(g)+c_{3} ; c_{1}, c_{2}, c_{3} \in \mathbb{R}\right\},
$$

and linear functional $M$ on $\mathbb{X}$ defined by

$$
M(h)=\alpha h(a)+\beta h(b)+\gamma \sum_{j=1}^{m} \gamma_{j} h\left(x_{j}\right) .
$$

The functional $M$ is unital since $\alpha+\beta+\gamma \sum_{j=1}^{m} \gamma_{j}=1$ and positive since the function $h=\left(c_{1}+c_{2} f\right)(g)+c_{3}$ is monotone.

If $f$ is not monotone, then we translate $f$ so that its minimum $f\left(x_{0}\right)=0$ and express $f=f_{1}+f_{2}$ where

$$
\begin{aligned}
& f_{1}(x)= \begin{cases}0 & \text { for } x \in\left[c, x_{0}\right] \\
f(x) & \text { for } x \in\left[x_{0}, d\right]\end{cases} \\
& f_{2}(x)= \begin{cases}f(x) & \text { for } x \in\left[c, x_{0}\right] \\
0 & \text { for } x \in\left[x_{0}, d\right]\end{cases}
\end{aligned}
$$

with $[c, d]=\operatorname{conv}\{g(a), g(b)\}$. The previous procedure should be applied to the monotone convex functions $f_{1}$ and $f_{2}$.

Putting the identity function $g(x)=x$ for $x \in[a, b]$, the inequality in (19) is reduced to

$$
\begin{aligned}
& f\left(\alpha a+\beta b+\gamma \sum_{j=1}^{m} \gamma_{j} x_{j}\right) \\
& \leq \alpha f(a)+\beta f(b)+\gamma \sum_{j=1}^{m} \gamma_{j} f\left(x_{j}\right) .
\end{aligned}
$$

If $\alpha=\beta=0$ and $\gamma=1$, the above inequality represents the Jensen inequality. If $\alpha=\beta=1$ and $\gamma=-1$, it represents the Jensen-Mercer inequality.

3.2. Inequalities for Convex Functions with Several Variables. We assume that $\mathbb{R}^{n}$ is the real vector space treating its points as vectors with the standard coordinate addition $\left(x_{1}, \ldots, x_{n}\right)+$ $\left(y_{1}, \ldots, y_{n}\right)=\left(x_{1}+y_{1}, \ldots, x_{n}+y_{n}\right)$ and scalar multiplication $\alpha\left(x_{1}, \ldots, x_{n}\right)=\left(\alpha x_{1}, \ldots, \alpha x_{n}\right)$. The aim of this subsection is to generalize Theorem 12 to simplexes.

To begin let us show how Lemma 9 can be generalized to the triangle $(n=2)$. Take the three planar points $A_{1}\left(x_{1}, y_{1}\right)$, $A_{2}\left(x_{2}, y_{2}\right)$, and $A_{3}\left(x_{3}, y_{3}\right)$ that do not belong to one line. Then any point $P(x, y) \in \mathbb{R}^{2}$ is presented by the unique affine combination

$$
P=\alpha_{P 1} A_{1}+\alpha_{P 2} A_{2}+\alpha_{P 3} A_{3}
$$

where

$$
\alpha_{P 1}=\frac{\left|\begin{array}{lll}
x & y & 1 \\
x_{2} & y_{2} & 1 \\
x_{3} & y_{3} & 1
\end{array}\right|}{\left|\begin{array}{lll}
x_{1} & y_{1} & 1 \\
x_{2} & y_{2} & 1 \\
x_{3} & y_{3} & 1
\end{array}\right|},
$$

$$
\alpha_{P 2}=-\frac{\left|\begin{array}{lll}
x & y & 1 \\
x_{1} & y_{1} & 1 \\
x_{3} & y_{3} & 1
\end{array}\right|}{\left|\begin{array}{lll}
x_{1} & y_{1} & 1 \\
x_{2} & y_{2} & 1 \\
x_{3} & y_{3} & 1
\end{array}\right|},
$$

$$
\alpha_{P 3}=\frac{\left|\begin{array}{lll}
x & y & 1 \\
x_{1} & y_{1} & 1 \\
x_{2} & y_{2} & 1
\end{array}\right|}{\left|\begin{array}{lll}
x_{1} & y_{1} & 1 \\
x_{2} & y_{2} & 1 \\
x_{3} & y_{3} & 1
\end{array}\right|} .
$$

So, we suppose that $\operatorname{conv}\left\{A_{1}, A_{2}, A_{3}\right\}$ is the triangle with vertices $A_{1}, A_{2}$, and $A_{3}$.

Lemma 13. Let $C \in \operatorname{conv}\left\{A_{1}, A_{2}, A_{3}\right\}$ be a point. Let $\alpha_{1}, \alpha_{2}$, $\alpha_{3} \in[0,1]$ and $\gamma \in[-1,1]$ be coefficients such that $\alpha_{1}, \alpha_{2}, \alpha_{3} \geq$ $-\gamma$ and $\alpha_{1}+\alpha_{2}+\alpha_{3}+\gamma=1$.

Then the affine combination $\alpha_{1} A_{1}+\alpha_{2} A_{2}+\alpha_{3} A_{3}+\gamma C \in$ $\operatorname{conv}\left\{A_{1}, A_{2}, A_{3}\right\}$.

Proof. Let us show that the affine combination $\alpha_{1} A_{1}+\alpha_{2} A_{2}+$ $\alpha_{3} A_{3}+\gamma C$ is actually the convex combination. Since the point $C \in \operatorname{conv}\left\{A_{1}, A_{2}, A_{3}\right\}$, the convex combination $C=\alpha_{C 1} A_{1}+$ $\alpha_{\mathrm{C} 2} A_{2}+\alpha_{\mathrm{C} 3} A_{3}$ holds with the coefficients $\alpha_{\mathrm{C} 1}, \alpha_{\mathrm{C} 2}$, and $\alpha_{\mathrm{C} 3}$ taken from the formulas in (25). Therefore, we have

$$
\begin{aligned}
\alpha_{1} A_{1} & +\alpha_{2} A_{2}+\alpha_{3} A_{3}+\gamma C \\
= & {\left[\alpha_{1}\left(1-\alpha_{C 1}\right)+\left(\alpha_{1}+\gamma\right) \alpha_{C 1}\right] A_{1} } \\
& +\left[\alpha_{2}\left(1-\alpha_{C 2}\right)+\left(\alpha_{2}+\gamma\right) \alpha_{C 2}\right] A_{2} \\
& +\left[\alpha_{3}\left(1-\alpha_{C 3}\right)+\left(\alpha_{3}+\gamma\right) \alpha_{C 3}\right] A_{3} .
\end{aligned}
$$

The coefficients in the square brackets are nonnegative with the sum equal to 1 , so the observed combination is convex, and its center $A=\alpha_{1} A_{1}+\alpha_{2} A_{2}+\alpha_{3} A_{3}+\gamma C$ belongs to $\operatorname{conv}\left\{A_{1}, A_{2}, A_{3}\right\}$.

If $A_{1}, \ldots, A_{n+1} \in \mathbb{R}^{n}$ are points such that the points $A_{1}-$ $A_{n+1}, \ldots, A_{n}-A_{n+1}$ are linearly independent, then the convex hull $\operatorname{conv}\left\{A_{1}, \ldots, A_{n+1}\right\}$ is called the $n$-simplex with vertices $A_{1}, \ldots, A_{n+1}$. Geometrically speaking, all the simplex vertices cannot belong to the same hyperplane. If $A_{k}=$ $A_{k}\left(x_{k 1}, \ldots, x_{k n}\right)$, then any point $P\left(x_{1}, \ldots, x_{n}\right) \in \mathbb{R}^{n}$ is presented by the unique affine combination

$$
P=\sum_{k=1}^{n+1} \alpha_{P k} A_{k}
$$


where the numerators $\alpha_{P k}^{\text {num }}$ and denominators $\alpha_{P k}^{\text {den }}$ of the coefficients $\alpha_{P k}$ are

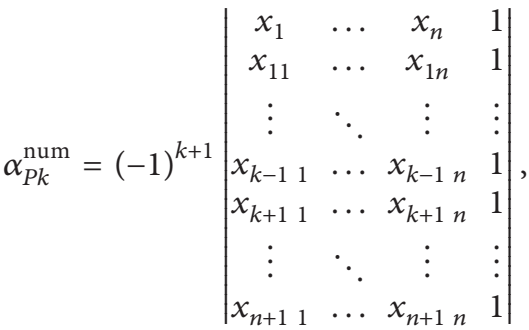

$$
\begin{aligned}
& \alpha_{P k}^{\mathrm{den}}=\left|\begin{array}{cccc}
x_{11} & \ldots & x_{1 n} & 1 \\
\vdots & \ddots & \vdots & \vdots \\
x_{k-1} & \ldots & x_{k-1 n} & 1 \\
x_{k 1} & \ldots & x_{k n} & 1 \\
x_{k+1} & \ldots & x_{k+1} n & 1 \\
\vdots & \ddots & \vdots & \vdots \\
x_{n+1} & \ldots & x_{n+1} n & 1
\end{array}\right| .
\end{aligned}
$$

In what follows we assume that $\operatorname{conv}\left\{A_{1}, \ldots, A_{n+1}\right\} \subset \mathbb{R}^{n}$ is the $n$-simplex with vertices $A_{1}, \ldots, A_{n+1}$.

The generalization of Lemma 13 (in $n$ dimensions) to several points $C_{j}$ stands as follows.

Theorem 14. Let $C_{j} \in \operatorname{conv}\left\{A_{1}, \ldots, A_{n+1}\right\}$ be points for $j=$ $1, \ldots, m$. Let $\alpha_{k}, \gamma_{j} \in[0,1]$ and $\gamma \in[-1,1]$ be coefficients such that all $\alpha_{k} \geq-\gamma$ and $\sum_{k=1}^{n+1} \alpha_{k}+\gamma=\sum_{j=1}^{m} \gamma_{j}=1$.

Then the affine combination

$$
\sum_{k=1}^{n+1} \alpha_{k} A_{k}+\gamma \sum_{j=1}^{m} \gamma_{j} C_{j} \in \operatorname{conv}\left\{A_{1}, \ldots, A_{n+1}\right\}
$$

Corollary 15. Let $g: \operatorname{conv}\left\{A_{1}, \ldots, A_{n+1}\right\} \rightarrow \mathbb{R}^{n}$ be a monotone mapping (either nondecreasing in each variable or nonincreasing in each variable), and let $P_{j} \in \operatorname{conv}\left\{A_{1}, \ldots\right.$, $\left.A_{n+1}\right\}$ be points for $j=1, \ldots, m$. Let $\alpha_{k}, \gamma_{j} \in[0,1]$ and $\gamma \in$ $[-1,1]$ be coefficients such that all $\alpha_{k} \geq-\gamma$ and $\sum_{k=1}^{n+1} \alpha_{k}+\gamma=$ $\sum_{j=1}^{m} \gamma_{j}=1$.

Then the affine combination

$$
\sum_{k=1}^{n+1} \alpha_{k} g\left(A_{k}\right)+\gamma \sum_{j=1}^{m} \gamma_{j} g\left(P_{j}\right) \in \operatorname{conv}\left\{g\left(A_{1}\right), \ldots, g\left(A_{n+1}\right)\right\} .
$$

Theorem 16. Let $g: \operatorname{conv}\left\{A_{1}, \ldots, A_{n+1}\right\} \rightarrow \mathbb{R}^{n}$ be a monotone mapping (either nondecreasing in each variable or nonincreasing in each variable), and let $P_{j} \in \operatorname{conv}\left\{A_{1}, \ldots, A_{n+1}\right\}$ be points for $j=1, \ldots, m$. Let $\alpha_{k}, \gamma_{j} \in[0,1]$ and $\gamma \in[-1,1]$ be coefficients such that all $\alpha_{k} \geq-\gamma$ and $\sum_{k=1}^{n+1} \alpha_{k}+\gamma=\sum_{j=1}^{m} \gamma_{j}=1$.
Then every continuous convex function $f: \operatorname{conv}\left\{g\left(A_{1}\right)\right.$, $\left.\ldots, g\left(A_{n+1}\right)\right\} \rightarrow \mathbb{R}$ satisfies the inequality

$$
\begin{aligned}
& f\left(\sum_{k=1}^{n+1} \alpha_{k} g\left(A_{k}\right)+\gamma \sum_{j=1}^{m} \gamma_{j} g\left(P_{j}\right)\right) \\
& \quad \leq \sum_{k=1}^{n+1} \alpha_{k} f\left(g\left(A_{k}\right)\right)+\gamma \sum_{j=1}^{m} \gamma_{j} f\left(g\left(P_{j}\right)\right) .
\end{aligned}
$$

Proof. Let us prove the case $n=2$.

If $f$ is monotone (either nondecreasing in both variables or nonincreasing in both variables), then we apply the McShane inequality in (3) to the function space

$$
\begin{gathered}
\mathbb{X}=\left\{h: \operatorname{conv}\left\{A_{1}, A_{2}, A_{3}\right\} \rightarrow \mathbb{R} \mid h=c_{1} g\right. \\
\left.+c_{2} f(g)+c_{3} ; c_{1}, c_{2}, c_{3} \in \mathbb{R}\right\}
\end{gathered}
$$

and linear functional $M$ on $\mathbb{X}$ defined by

$$
M(h)=\sum_{k=1}^{3} \alpha_{k} h\left(A_{k}\right)+\gamma \sum_{j=1}^{m} \gamma_{j} h\left(P_{j}\right) .
$$

The functional $M$ is unital since $\sum_{k=1}^{3} \alpha_{k}+\gamma \sum_{j=1}^{m} \gamma_{j}=1$ and positive since the function $h=\left(c_{1}+c_{2} f\right)(g)+c_{3}$ is monotone.

If $f$ is not monotone, then we translate $f$ so that its minimum $f\left(x_{0}, y_{0}\right)=0$ and divide the triangle $\operatorname{conv}\left\{g\left(A_{1}\right)\right.$, $\left.g\left(A_{2}\right), g\left(A_{3}\right)\right\}$ into $2^{2}=4$ parts considering the lines $x=x_{0}$ and $y=y_{0}$. Then we express $f$ as the sum of the monotone convex functions, $f=f_{1}+f_{2}+f_{3}+f_{4}$, and apply the previous procedure to these functions.

The proof goes in the same way for any integer $n$.

Applying the identity mapping $g(P)=P$ for $P \in \operatorname{conv}\left\{A_{1}\right.$, $\left.\ldots, A_{n+1}\right\}$ to the inequality in (31), it reads as follows:

$$
\begin{aligned}
& f\left(\sum_{k=1}^{n+1} \alpha_{k} A_{k}+\gamma \sum_{j=1}^{m} \gamma_{j} P_{j}\right) \\
& \quad \leq \sum_{k=1}^{n+1} \alpha_{k} f\left(A_{k}\right)+\gamma \sum_{j=1}^{m} \gamma_{j} f\left(P_{j}\right) .
\end{aligned}
$$

\section{References}

[1] B. Jessen, "Bemrkninger om konvekse Funktioner og Uligheder imellem Middelvrdier. I," Matematisk Tidsskrift B, vol. 2, pp. 1728, 1931.

[2] J. Mićić, Z. Pavić, and J. Pečarić, "The inequalities for quasiarithmetic means," Abstract and Applied Analysis, vol. 2012, Article ID 203145, 25 pages, 2012.

[3] I. Raşa, "A note on Jessen's inequality," in Itinerant Seminar on Functional Equations, Approximation and Convexity, pp. 275280, Universitatea Babes-Bolyai, Cluj-Napoca, Romania, 1988.

[4] E. J. McShane, "Jensen's inequality," Bulletin of the American Mathematical Society, vol. 43, no. 8, pp. 521-527, 1937. 


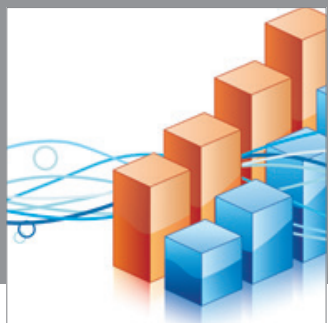

Advances in

Operations Research

mansans

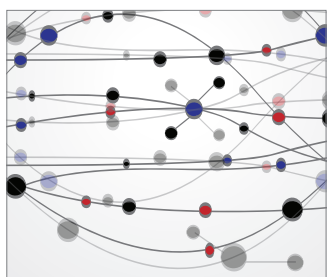

The Scientific World Journal
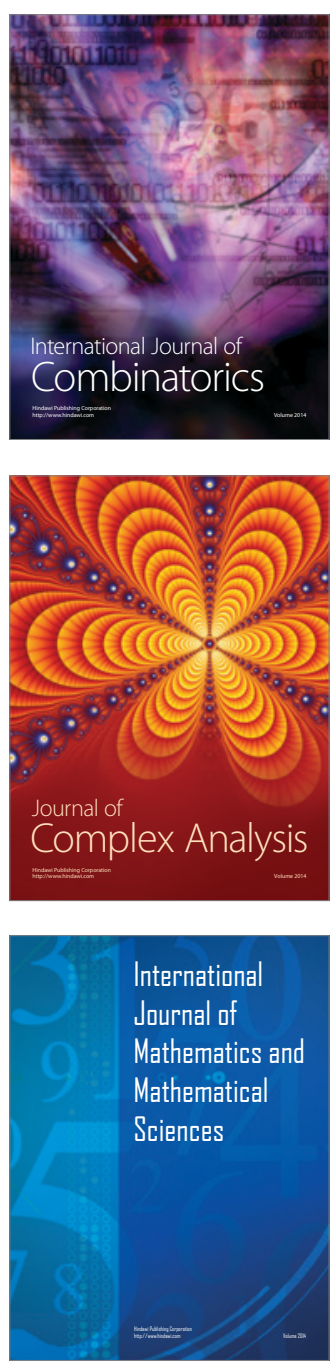
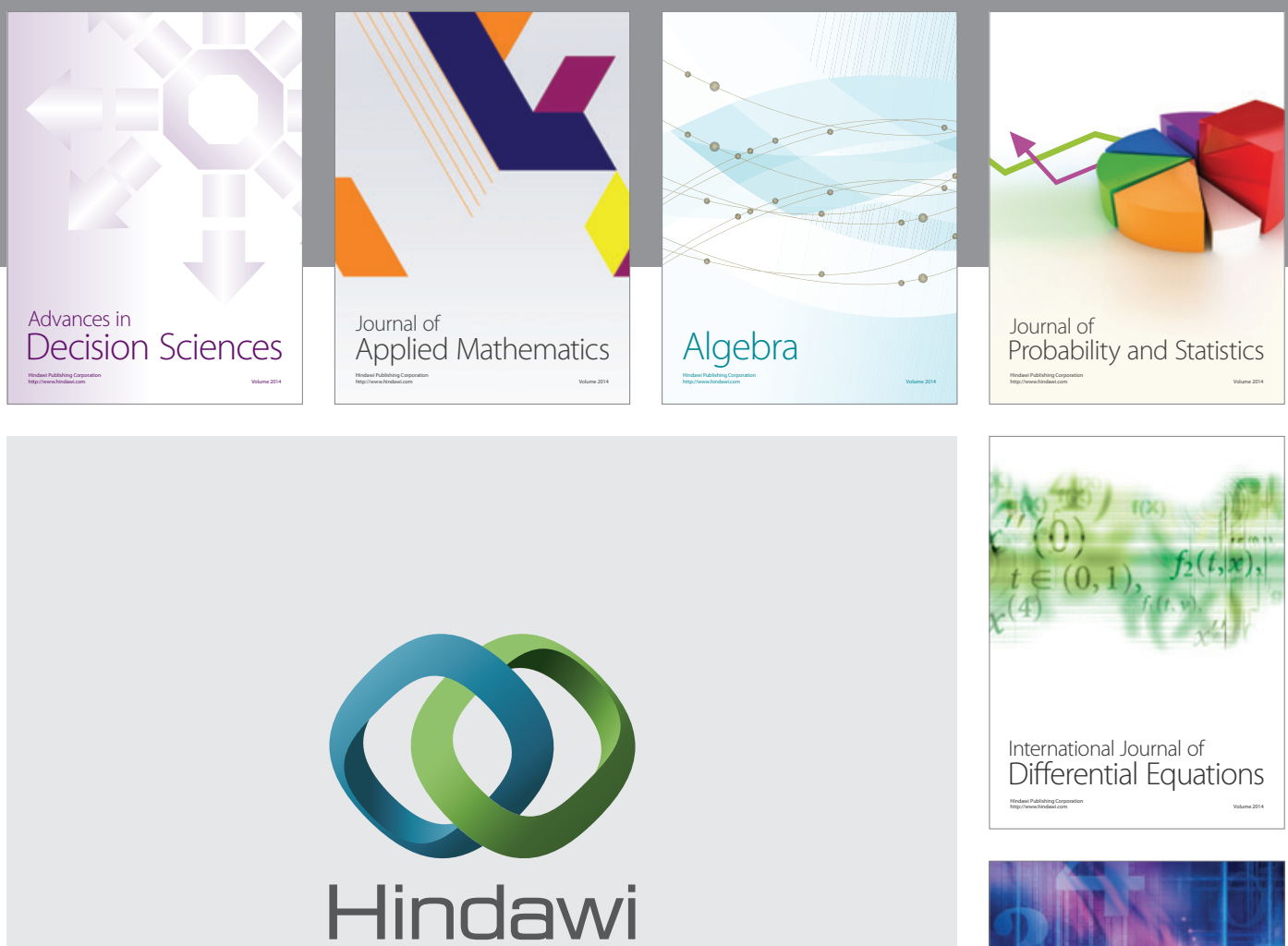

Submit your manuscripts at http://www.hindawi.com
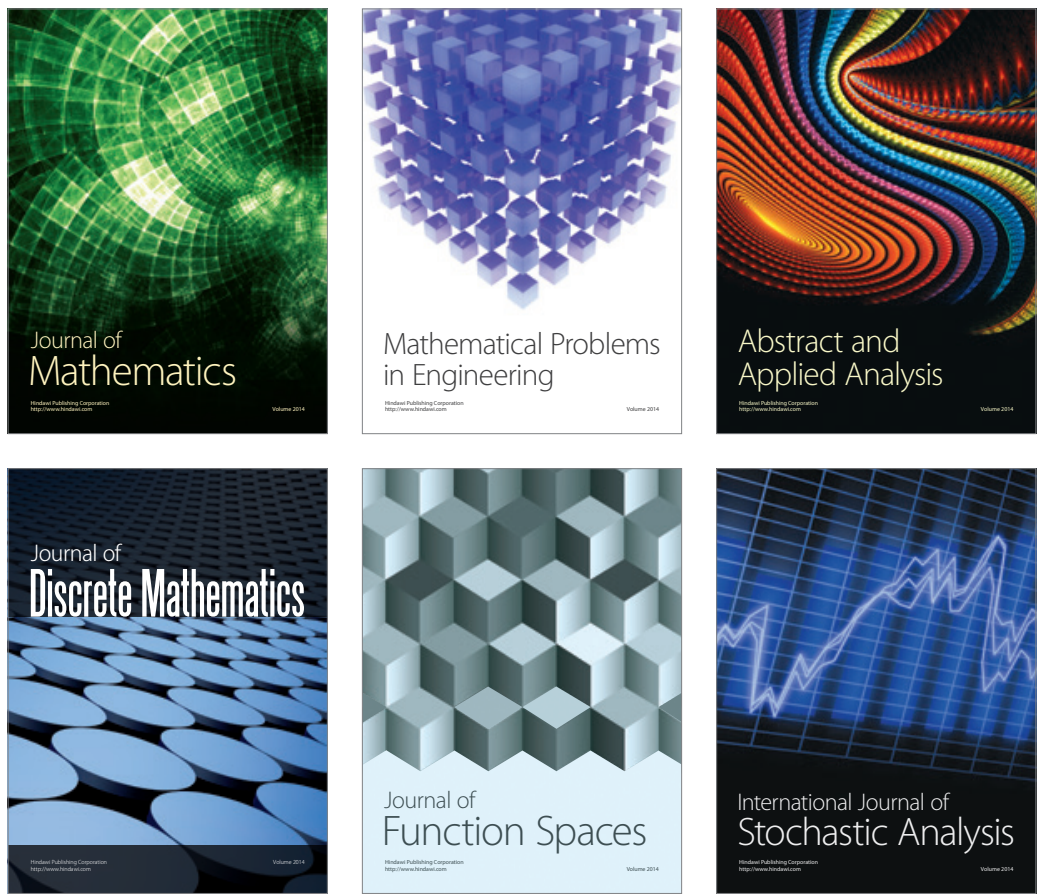

Journal of

Function Spaces

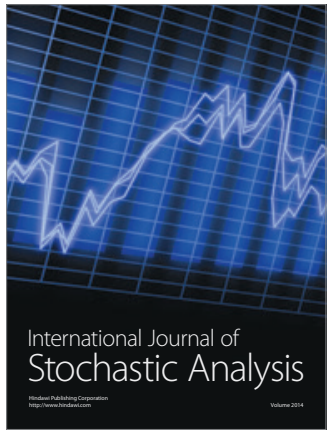

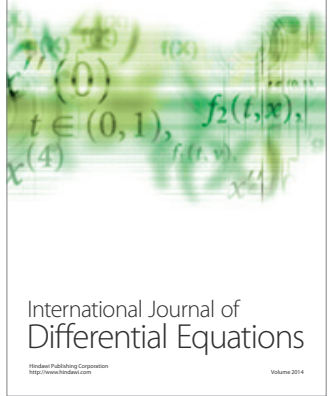
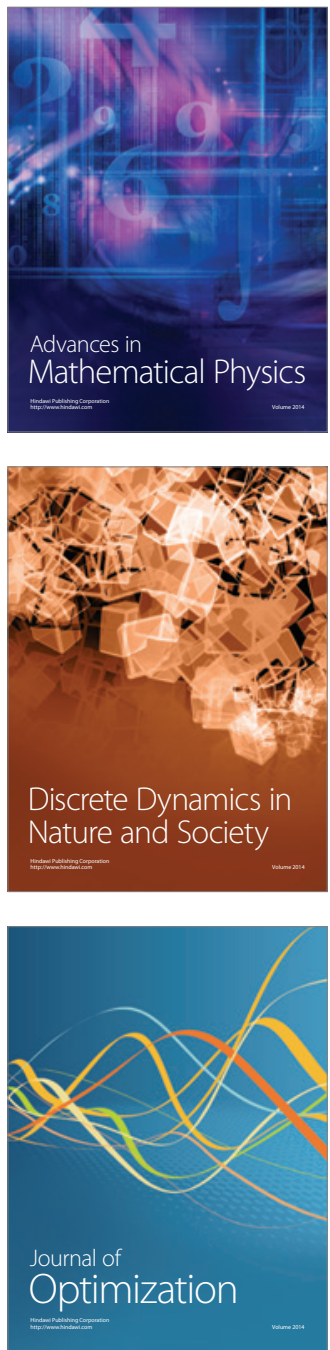